\title{
The Voronovskaja type theorem for a general class of Szász-Mirakjan operators
}

\author{
Ovidiu T. Pop, Dan Miclăus, and Dan Bărbosu
}




\title{
THE VORONOVSKAJA TYPE THEOREM FOR A GENERAL CLASS OF SZÁSZ-MIRAKJAN OPERATORS
}

\author{
OVIDIU T. POP, DAN MICLĂUŞ, AND DAN BĂRBOSU
}

Received June 8, 2011

\begin{abstract}
The paper is devoted to defining a new general class of linear and positive operators depending on a certain function $\varphi$. This new class of linear and positive operators generalizes the Szász-Mirakjan operators. For this new class of operators we establish a convergence theorem and the evaluation of the rate of convergence, in terms of the modulus of continuity.
\end{abstract}

2000 Mathematics Subject Classification: 41A10; 41A25; 41A36

Keywords: Voronovskaja's type theorem, Szász-Mirakjan operators, positivity, linearity, modulus of continuity

\section{INTRODUCTION}

Let $\mathbb{N}$ be the set of positive integers and $\mathbb{N}_{0}=\mathbb{N} \cup\{0\}$. In this section we recall some results from [12], which we shall use in the present paper.

Let $I, J$ be real intervals and $I \cap J \neq \varnothing$. For any $n, k \in \mathbb{N}_{0}, n \neq 0$ consider the functions $\varphi_{n, k}: J \rightarrow \mathbb{R}$, with the property that $\varphi_{n, k}(x) \geq 0$, for any $x \in J$ and the linear positive functionals $A_{n, k}: E(I) \rightarrow \mathbb{R}$.

For any $n \in \mathbb{N}$ define the operator $L_{n}: E(I) \rightarrow F(J)$, by

$$
\left(L_{n} f\right)(x)=\sum_{k=0}^{\infty} \varphi_{n, k}(x) A_{n, k}(f),
$$

where $E(I)$ is a linear space of real-valued functions defined on $I$, for which the operators (1.1) are convergent and $F(J)$ is a subset of the set of real-valued functions defined on $J$.

Remark 1. [12] The operators $\left(L_{n}\right)_{n \in \mathbb{N}}$ are linear and positive on $E(I \cap J)$.

For $n, i \in \mathbb{N}_{0}, n \neq 0$, let the function $\psi_{x}$ be defined by $\psi_{x}(t)=t-x,(x, t) \in$ $\left[0,+\infty\left[\times\left[0,+\infty\left[\right.\right.\right.\right.$ and define $T_{n, i}^{*}$ by

$$
\left(T_{n, i}^{*} L_{n}\right)(x)=n^{i}\left(L_{n} \psi_{x}^{i}\right)(x)=n^{i} \sum_{k=0}^{\infty} \varphi_{n, k}(x) A_{n, k}\left(\psi_{x}^{i}\right), x \in I \cap J .
$$


In what follows $s \in \mathbb{N}_{0}$ is even and we suppose that the following two conditions hold:

- there exists the smallest $\alpha_{s}, \alpha_{s+2} \in[0,+\infty[$, so that

$$
\lim _{n \rightarrow \infty} \frac{\left(T_{n, j}^{*} L_{n}\right)(x)}{n^{\alpha_{j}}}=B_{j}(x) \in \mathbb{R},
$$

for any $x \in I \cap J$ and $j \in\{s, s+2\}$,

$$
\alpha_{s+2}<\alpha_{s}+2
$$

- $I \cap J$ is an interval.

Theorem 1 ([12]). Let $f \in E(I)$ be a function. If $x \in I \cap J$ and $f$ is $s$ times differentiable in a neighborhood of $x, f^{(s)}$ is continuous in $x$, then

$$
\lim _{n \rightarrow \infty} n^{s-\alpha_{s}}\left(\left(L_{n} f\right)(x)-\sum_{i=0}^{s} \frac{f^{(i)}(x)}{n^{i} i !}\left(T_{n, i}^{*} L_{n}\right)(x)\right)=0 .
$$

Assume that $f$ is $s$ times differentiable on $I$ and there exists an interval $K \subset I \cap J$ such that, there exists $n(s) \in \mathbb{N}$ and the constants $k_{j} \in \mathbb{R}$ depending on $K$, so that for $n \geq n(s)$ and $x \in K$, the following

$$
\frac{\left(T_{n, j}^{*} L_{n}\right)(x)}{n^{\alpha_{j}}} \leq k_{j}
$$

holds, for $j \in\{s, s+2\}$.

Then, the convergence expressed by (1.5) is uniform on $K$ and

$$
\begin{aligned}
& n^{s-\alpha_{s}}\left|\left(L_{n} f\right)(x)-\sum_{i=0}^{s} \frac{f^{(i)}(x)}{n^{i} i !}\left(T_{n, i}^{*} L_{n}\right)(x)\right| \\
& \quad \leq \frac{1}{s !}\left(k_{s}+k_{s+2}\right) \omega_{1}\left(f^{(s)} ; \frac{1}{\sqrt{n^{2+\alpha_{s}-\alpha_{s+2}}}}\right),
\end{aligned}
$$

for any $x \in K, n \geq n(s)$, where $\omega_{1}(f ; \delta)$ denotes the modulus of continuity [1], (the first order modulus of smoothness) of the function $f$.

In [11], C. Mortici defined the sequence of operators

$$
\varphi S_{n}: C^{2}\left(\left[0,+\infty[) \rightarrow C^{\infty}([0,+\infty[)\right.\right.
$$

given by

$$
\left(\varphi S_{n} f\right)(x)=\frac{1}{\varphi(n x)} \sum_{k=0}^{\infty} \frac{\varphi^{(k)}(0)}{k !}(n x)^{k} f\left(\frac{k}{n}\right),
$$

for any $x \in[0,+\infty[$ and $n \in \mathbb{N}$, where $\varphi: \mathbb{R} \rightarrow] 0,+\infty[$ is an analytic function. These are called the $\varphi$-Szász-Mirakjan operators, because in the case when $\varphi(y)=e^{y}$, they reduce to the classical Mirakjan-Favard-Szász operators [5, 10] and [15]. 
Remark 2. Similar generalization of this type are the operators defined and studied by Jakimovski and Leviatan [7] or the operators defined by Baskakov in 1957 (see, e.g., the book [2], subsection 5.3.11, p. 344, where they are attributed to Mastroianni). Another recently similar generalization of this type are the operators defined and studied by authors in $[3,4,8,9]$ and [13].

Remark 3. The classical Mirakjan-Favard-Szász operators $S_{n}: C_{2}([0,+\infty[) \rightarrow$ $C([0,+\infty[)$ are defined by

$$
\left(S_{n} f\right)(x)=e^{-n x} \sum_{k=0}^{\infty} \frac{(n x)^{k}}{k !} f\left(\frac{k}{n}\right),
$$

where

$$
C_{2}\left(\left[0,+\infty[)=\left\{f \in C \left(\left[0,+\infty[): \lim _{x \rightarrow \infty} \frac{f(x)}{1+x^{2}} \text { exists and is finite }\right\} .\right.\right.\right.\right.
$$

In the following, we shall use the classical definition of Mirakjan-Favard-Szász operators, i.e., $f \in C_{2}([0,+\infty[)$.

The purpose of this paper is to introduce a new linear positive operators of SzászMirakjan type, in order to use it in the theory of uniform approximation of functions, which generalize some new, respectively older results, cited at the adequate moment. We shall prove uniform convergence, Voronovskaja type formulas and the order of approximation, for these new linear positive operators.

\section{Auxiliary Results}

We consider an analytic function $\varphi:[0,+\infty[\rightarrow] 0,+\infty[$ and it follows

$$
\varphi(x)=\sum_{k=0}^{\infty} \frac{\varphi^{(k)}(0)}{k !} x^{k} .
$$

By differentiation of the relation (2.1), we get

$$
\varphi^{(1)}(x)=\sum_{k=1}^{\infty} \frac{\varphi^{(k)}(0)}{(k-1) !} x^{k-1}
$$

and

$$
\varphi^{(2)}(x)=\sum_{k=2}^{\infty} \frac{\varphi^{(k)}(0)}{(k-2) !} x^{k-2} .
$$

We consider the function $\alpha:[0,+\infty[\rightarrow \mathbb{R}$ defined by

$$
\alpha(x)=\frac{\varphi^{(1)}(x)}{\varphi(x)}-1 \text {, }
$$

for any $x \in[0,+\infty[$. 
Remark 4. From (2.4) it follows that $\alpha$ is also an analytic function.

Let $\left(a_{n}\right)_{n \in \mathbb{N}}$ be a sequence, so that for any $n \in \mathbb{N}, a_{n}>0$ and we assume that the function $\varphi$ has the properties

$$
\lim _{x \rightarrow \infty} \frac{a_{n}}{n} \frac{\varphi^{(1)}\left(a_{n} x\right)}{\varphi\left(a_{n} x\right)}=1
$$

and

where $x \in[0,+\infty[$.

$$
\lim _{x \rightarrow \infty}\left(\frac{a_{n}}{n}\right)^{2} \frac{\varphi^{(2)}\left(a_{n} x\right)}{\varphi\left(a_{n} x\right)}=1,
$$

Lemma 1. The following identity

$$
\varphi(x)=c \cdot e^{x+\int_{0}^{x} \alpha(t) d t}
$$

holds, for any $x \in[0,+\infty[$, where $c>0$.

Proof. From (2.4), we get

$$
\varphi^{(1)}(x)-(1+\alpha(x)) \varphi(x)=0,
$$

for any $x \in[0,+\infty[$. This relation is equivalent to

$$
\left(\varphi(x) \cdot e^{-x-\int_{0}^{x} \alpha(t) d t}\right)^{(1)}=0
$$

for any $x \in[0,+\infty[$. Because $\varphi:[0,+\infty[\rightarrow] 0,+\infty[$ exists $c>0$, so that

$$
\varphi(x) \cdot e^{-x-\int_{0}^{x} \alpha(t) d t}=c,
$$

for any $x \in[0,+\infty[$.

The relation (2.7) follows immediately from the above equality.

\section{THE $\varphi$-SZÁSZ-MIRAKJAN TYPE OPERATORS}

We define the sequence of operators $\varphi S_{n}: C_{2}([0,+\infty[) \rightarrow C([0,+\infty[)$, given by

$$
\left(\varphi S_{n} f\right)(x)=\frac{1}{\varphi\left(a_{n} x\right)} \sum_{k=0}^{\infty} \frac{\varphi^{(k)}(0)}{k !}\left(a_{n} x\right)^{k} f\left(\frac{k}{n}\right),
$$

for any $x \in[0,+\infty[$ and $n \in \mathbb{N}$. The operators (3.1) are called the $\varphi$-Szász-Mirakjan type operators, because in the case when $a_{n}=n$, for any $n \in \mathbb{N}$, the $\varphi$-Szász-Mirakjan operators [11] are obtained. In the case when $a_{n}=n$, for any $n \in \mathbb{N}$ and $\varphi(x)=e^{x}$, for any $x \in[0,+\infty[$, the operators (3.1) become the classical Mirakjan-Favard-Szász operators. 
Lemma 2. Let $e_{j}(x)=x^{j}, j \in\{0,1,2\}$ be the test functions. The $\varphi$-SzászMirakjan type operators satisfy

i) $\left(\varphi S_{n} e_{0}\right)(x)=1$,

ii) $\left(\varphi S_{n} e_{1}\right)(x)=\frac{a_{n}}{n} \frac{\varphi^{(1)}\left(a_{n} x\right)}{\varphi\left(a_{n} x\right)} x$,

iii) $\left(\varphi S_{n} e_{2}\right)(x)=\left(\frac{a_{n}}{n}\right)^{2} \frac{\varphi^{(2)}\left(a_{n} x\right)}{\varphi\left(a_{n} x\right)} x^{2}+\frac{1}{n} \frac{a_{n}}{n} \frac{\varphi^{(1)}\left(a_{n} x\right)}{\varphi\left(a_{n} x\right)} x$, for any $x \in[0,+\infty[$ and $n \in \mathbb{N}$.

Proof. We get

$$
\left(\varphi S_{n} e_{0}\right)(x)=\frac{1}{\varphi\left(a_{n} x\right)} \sum_{k=0}^{\infty} \frac{\varphi^{(k)}(0)}{k !}\left(a_{n} x\right)^{k}=1,
$$

taking (2.1) into account.

Next, we get

$$
\begin{gathered}
\left(\varphi S_{n} e_{1}\right)(x)=\frac{1}{\varphi\left(a_{n} x\right)} \sum_{k=0}^{\infty} \frac{\varphi^{(k)}(0)}{k !}\left(a_{n} x\right)^{k} \frac{k}{n} \\
=\frac{a_{n}}{n} \frac{x}{\varphi\left(a_{n} x\right)} \sum_{k=1}^{\infty} \frac{\varphi^{(k)}(0)}{(k-1) !}\left(a_{n} x\right)^{k-1}=\frac{a_{n}}{n} \frac{\varphi^{(1)}\left(a_{n} x\right)}{\varphi\left(a_{n} x\right)} x,
\end{gathered}
$$

taking (2.2) into account. In a similar way, one obtains

$$
\begin{gathered}
\left(\varphi S_{n} e_{2}\right)(x)=\frac{1}{\varphi\left(a_{n} x\right)} \sum_{k=0}^{\infty} \frac{\varphi^{(k)}(0)}{k !}\left(a_{n} x\right)^{k}\left(\frac{k}{n}\right)^{2} \\
=\frac{1}{n^{2}} \frac{1}{\varphi\left(a_{n} x\right)} \sum_{k=0}^{\infty} \frac{\varphi^{(k)}(0)}{k !}\left(a_{n} x\right)^{k}(k(k-1)+k) \\
=\left(\frac{a_{n}}{n}\right)^{2} \frac{x^{2}}{\varphi\left(a_{n} x\right)} \sum_{k=2}^{\infty} \frac{\varphi^{(k)}(0)}{(k-2) !}\left(a_{n} x\right)^{k-2}+\frac{a_{n}}{n^{2}} \frac{x}{\varphi\left(a_{n} x\right)} \sum_{k=1}^{\infty} \frac{\varphi^{(k)}(0)}{(k-1) !}\left(a_{n} x\right)^{k-1} \\
=\left(\frac{a_{n}}{n}\right)^{2} \frac{\varphi^{(2)}\left(a_{n} x\right)}{\varphi\left(a_{n} x\right)} x^{2}+\frac{1}{n} \frac{a_{n}}{n} \frac{\varphi^{(1)}\left(a_{n} x\right)}{\varphi\left(a_{n} x\right)} x,
\end{gathered}
$$

taking (2.2) and (2.3) into account. 
Lemma 3. Let $e_{j}(x)=x^{j}, j \in\{3,4\}$ be the test functions. The $\varphi$-Szász-Mirakjan type operators satisfy

$$
\begin{gathered}
\left(\varphi S_{n} e_{3}\right)(x)=\left(\frac{a_{n}}{n}\right)^{3} \frac{\varphi^{(3)}\left(a_{n} x\right)}{\varphi\left(a_{n} x\right)} x^{3}+\frac{3}{n}\left(\frac{a_{n}}{n}\right)^{2} \frac{\varphi^{(2)}\left(a_{n} x\right)}{\varphi\left(a_{n} x\right)} x^{2} \\
+\frac{1}{n^{2}} \frac{a_{n}}{n} \frac{\varphi^{(1)}\left(a_{n} x\right)}{\varphi\left(a_{n} x\right)} x
\end{gathered}
$$

and

$$
\begin{gathered}
\left(\varphi S_{n} e_{4}\right)(x)=\left(\frac{a_{n}}{n}\right)^{4} \frac{\varphi^{(4)}\left(a_{n} x\right)}{\varphi\left(a_{n} x\right)} x^{4}+\frac{6}{n}\left(\frac{a_{n}}{n}\right)^{3} \frac{\varphi^{(3)}\left(a_{n} x\right)}{\varphi\left(a_{n} x\right)} x^{3} \\
+\frac{7}{n^{2}}\left(\frac{a_{n}}{n}\right)^{2} \frac{\varphi^{(2)}\left(a_{n} x\right)}{\varphi\left(a_{n} x\right)} x^{2}+\frac{1}{n^{3}} \frac{a_{n}}{n} \frac{\varphi^{(1)}\left(a_{n} x\right)}{\varphi\left(a_{n} x\right)} x,
\end{gathered}
$$

for any $x \in[0,+\infty[$ and $n \in \mathbb{N}$.

Proof. Because the function $\varphi$ is analytic by differentiation, it follows

$$
\varphi^{(3)}(x)=\sum_{k=3}^{\infty} \frac{\varphi^{(k)}(0)}{(k-3) !} x^{k-3}, \varphi^{(4)}(x)=\sum_{k=4}^{\infty} \frac{\varphi^{(k)}(0)}{(k-4) !} x^{k-4} .
$$

Using the above relations and (2.1), (2.2), then from (3.1), it follows

$$
\begin{aligned}
& \left(\varphi S_{n} e_{3}\right)(x)=\frac{1}{\varphi\left(a_{n} x\right)} \sum_{k=0}^{\infty} \frac{\varphi^{(k)}(0)}{k !}\left(a_{n} x\right)^{k}\left(\frac{k}{n}\right)^{3} \\
& \quad=\frac{1}{n^{3}} \frac{1}{\varphi\left(a_{n} x\right)} \sum_{k=0}^{\infty} \frac{\varphi^{(k)}(0)}{k !}\left(a_{n} x\right)^{k} \cdot u_{1}(k) \\
& =\left(\frac{a_{n}}{n}\right)^{3} \frac{x^{3}}{\varphi\left(a_{n} x\right)} \sum_{k=3}^{\infty} \frac{\varphi^{(k)}(0)}{(k-3) !}\left(a_{n} x\right)^{k-3} \\
& +\frac{3}{n}\left(\frac{a_{n}}{n}\right)^{2} \frac{x^{2}}{\varphi\left(a_{n} x\right)} \sum_{k=2}^{\infty} \frac{\varphi^{(k)}(0)}{(k-2) !}\left(a_{n} x\right)^{k-2}+\frac{1}{n^{2}} \frac{a_{n}}{n} \frac{x}{\varphi\left(a_{n} x\right)} \sum_{k=1}^{\infty} \frac{\varphi^{(k)}(0)}{(k-1) !}\left(a_{n} x\right)^{k-1} \\
& =\left(\frac{a_{n}}{n}\right)^{3} \frac{\varphi^{(3)}\left(a_{n} x\right)}{\varphi\left(a_{n} x\right)} x^{3}+\frac{3}{n}\left(\frac{a_{n}}{n}\right)^{2} \frac{\varphi^{(2)}\left(a_{n} x\right)}{\varphi\left(a_{n} x\right)} x^{2}+\frac{1}{n^{2}} \frac{a_{n}}{n} \frac{\varphi^{(1)}\left(a_{n} x\right)}{\varphi\left(a_{n} x\right)} x,
\end{aligned}
$$

where $u_{1}(k)=k(k-1)(k-2)+3 k(k-1)+k$.

$$
\begin{aligned}
& \left(\varphi S_{n} e_{4}\right)(x)=\frac{1}{\varphi\left(a_{n} x\right)} \sum_{k=0}^{\infty} \frac{\varphi^{(k)}(0)}{k !}\left(a_{n} x\right)^{k}\left(\frac{k}{n}\right)^{4} \\
& =\frac{1}{n^{4}} \frac{1}{\varphi\left(a_{n} x\right)} \sum_{k=0}^{\infty} \frac{\varphi^{(k)}(0)}{k !}\left(a_{n} x\right)^{k} \cdot u_{2}(k)
\end{aligned}
$$




$$
\begin{aligned}
& =\left(\frac{a_{n}}{n}\right)^{4} \frac{x^{4}}{\varphi\left(a_{n} x\right)} \sum_{k=4}^{\infty} \frac{\varphi^{(k)}(0)}{(k-4) !}\left(a_{n} x\right)^{k-4}+\frac{6}{n}\left(\frac{a_{n}}{n}\right)^{3} \frac{x^{3}}{\varphi\left(a_{n} x\right)} \sum_{k=3}^{\infty} \frac{\varphi^{(k)}(0)}{(k-3) !}\left(a_{n} x\right)^{k-3} \\
& +\frac{7}{n^{2}}\left(\frac{a_{n}}{n}\right)^{2} \frac{x^{2}}{\varphi\left(a_{n} x\right)} \sum_{k=2}^{\infty} \frac{\varphi^{(k)}(0)}{(k-2) !}\left(a_{n} x\right)^{k-2}+\frac{1}{n^{3}} \frac{a_{n}}{n} \frac{x}{\varphi\left(a_{n} x\right)} \sum_{k=1}^{\infty} \frac{\varphi^{(k)}(0)}{(k-1) !}\left(a_{n} x\right)^{k-1} \\
& =\left(\frac{a_{n}}{n}\right)^{4} \frac{\varphi^{(4)}\left(a_{n} x\right)}{\varphi\left(a_{n} x\right)} x^{4}+\frac{6}{n}\left(\frac{a_{n}}{n}\right)^{3} \frac{\varphi^{(3)}\left(a_{n} x\right)}{\varphi\left(a_{n} x\right)} x^{3}+\frac{7}{n^{2}}\left(\frac{a_{n}}{n}\right)^{2} \frac{\varphi^{(2)}\left(a_{n} x\right)}{\varphi\left(a_{n} x\right)} x^{2} \\
& +\frac{1}{n^{3}} \frac{a_{n}}{n} \frac{\varphi^{(1)}\left(a_{n} x\right)}{\varphi\left(a_{n} x\right)} x \\
& \text { where } u_{2}(k)=k(k-1)(k-2)(k-3)+6 k(k-1)(k-2)+7 k(k-1)+k
\end{aligned}
$$

Lemma 4. For any $x \in[0,+\infty[$ and $n \in \mathbb{N}$, the following

$$
\begin{gathered}
\left(T_{n, 0}^{*} \varphi S_{n}\right)(x)=1, \\
\left(T_{n, 1}^{*} \varphi S_{n}\right)(x)=n\left(\frac{a_{n}}{n} \frac{\varphi^{(1)}\left(a_{n} x\right)}{\varphi\left(a_{n} x\right)}-1\right) x, \\
\left(T_{n, 2}^{*} \varphi S_{n}\right)(x)=n^{2}\left(\left(\left(\frac{a_{n}}{n}\right)^{2} \frac{\varphi^{(2)}\left(a_{n} x\right)}{\varphi\left(a_{n} x\right)}-2 \frac{a_{n}}{n} \frac{\varphi^{(1)}\left(a_{n} x\right)}{\varphi\left(a_{n} x\right)}+1\right) x^{2}\right. \\
\left.+\frac{1}{n} \frac{a_{n}}{n} \frac{\varphi^{(1)}\left(a_{n} x\right)}{\varphi\left(a_{n} x\right)} x\right), \\
\left.+6\left(\frac{a_{n}}{n}\right)^{2} \frac{\varphi^{(2)}\left(a_{n} x\right)}{\varphi\left(a_{n} x\right)}-4 \frac{a_{n}}{n} \frac{\varphi^{(1)}\left(a_{n} x\right)}{\varphi\left(a_{n} x\right)}+1\right) x^{4} \\
+\frac{6}{n}\left(\left(\frac{a_{n}}{n}\right)^{3} \frac{\varphi^{(3)}\left(a_{n} x\right)}{\varphi\left(a_{n} x\right)}-2\left(\frac{a_{n}}{n}\right)^{2} \frac{\varphi^{(2)}\left(a_{n} x\right)}{\varphi\left(a_{n} x\right)}+\frac{a_{n}}{n} \frac{\varphi^{(1)}\left(a_{n} x\right)}{\varphi\left(a_{n} x\right)}\right) x^{3} \\
\left.+\frac{1}{n^{2}}\left(7\left(\frac{a_{n}}{n}\right)^{2} \frac{\varphi^{(2)}\left(a_{n} x\right)}{\varphi\left(a_{n} x\right)}-4 \frac{a_{n}}{n} \frac{\varphi^{(1)}\left(a_{n} x\right)}{\varphi\left(a_{n} x\right)}\right) x^{2}+\frac{1}{n^{3}} \frac{a_{n}}{n} \frac{\varphi^{(1)}\left(a_{n} x\right)}{\varphi\left(a_{n} x\right)} x\right)
\end{gathered}
$$

hold.

Proof. Taking into account relations (1.2), (3.1), Lemma 2 and Lemma 3, we get

$$
\begin{gathered}
\left(T_{n, 0}^{*} \varphi S_{n}\right)(x)=\left(\varphi S_{n} e_{0}\right)(x)=1, \\
\left(T_{n, 1}^{*} \varphi S_{n}\right)(x)=n\left(\varphi S_{n} \psi_{x}\right)(x)=n\left(\left(\varphi S_{n} e_{1}\right)(x)-x\left(\varphi S_{n} e_{0}\right)(x)\right)
\end{gathered}
$$




$$
\begin{aligned}
& =n\left(\frac{a_{n}}{n} \frac{\varphi^{(1)}\left(a_{n} x\right)}{\varphi\left(a_{n} x\right)}-1\right) x, \\
& \left(T_{n, 2}^{*} \varphi S_{n}\right)(x)=n^{2}\left(\varphi S_{n} \psi_{x}^{2}\right)(x)=n^{2}\left(\left(\varphi S_{n} e_{2}\right)(x)-2 x\left(\varphi S_{n} e_{1}\right)(x)+x^{2}\left(\varphi S_{n} e_{0}\right)(x)\right) \\
& =n^{2}\left(\left(\left(\frac{a_{n}}{n}\right)^{2} \frac{\varphi^{(2)}\left(a_{n} x\right)}{\varphi\left(a_{n} x\right)}-2 \frac{a_{n}}{n} \frac{\varphi^{(1)}\left(a_{n} x\right)}{\varphi\left(a_{n} x\right)}+1\right) x^{2}+\frac{1}{n} \frac{a_{n}}{n} \frac{\varphi^{(1)}\left(a_{n} x\right)}{\varphi\left(a_{n} x\right)} x\right), \\
& \left(T_{n, 4}^{*} \varphi S_{n}\right)(x)=n^{4}\left(\varphi S_{n} \psi_{x}^{4}\right)(x)=n^{4}\left(\left(\varphi S_{n} e_{4}\right)(x)-4 x\left(\varphi S_{n} e_{3}\right)(x)\right. \\
& \left.+6 x^{2}\left(\varphi S_{n} e_{2}\right)(x)-4 x^{3}\left(\varphi S_{n} e_{1}\right)(x)+x^{4}\left(\varphi S_{n} e_{0}\right)(x)\right) \\
& =n^{4}\left(\left(\left(\frac{a_{n}}{n}\right)^{4} \frac{\varphi^{(4)}\left(a_{n} x\right)}{\varphi\left(a_{n} x\right)}-4\left(\frac{a_{n}}{n}\right)^{3} \frac{\varphi^{(3)}\left(a_{n} x\right)}{\varphi\left(a_{n} x\right)}\right.\right. \\
& \left.+6\left(\frac{a_{n}}{n}\right)^{2} \frac{\varphi^{(2)}\left(a_{n} x\right)}{\varphi\left(a_{n} x\right)}-4 \frac{a_{n}}{n} \frac{\varphi^{(1)}\left(a_{n} x\right)}{\varphi\left(a_{n} x\right)}+1\right) x^{4} \\
& +\frac{6}{n}\left(\left(\frac{a_{n}}{n}\right)^{3} \frac{\varphi^{(3)}\left(a_{n} x\right)}{\varphi\left(a_{n} x\right)}-2\left(\frac{a_{n}}{n}\right)^{2} \frac{\varphi^{(2)}\left(a_{n} x\right)}{\varphi\left(a_{n} x\right)}+\frac{a_{n}}{n} \frac{\varphi^{(1)}\left(a_{n} x\right)}{\varphi\left(a_{n} x\right)}\right) x^{3} \\
& \left.+\frac{1}{n^{2}}\left(7\left(\frac{a_{n}}{n}\right)^{2} \frac{\varphi^{(2)}\left(a_{n} x\right)}{\varphi\left(a_{n} x\right)}-4 \frac{a_{n}}{n} \frac{\varphi^{(1)}\left(a_{n} x\right)}{\varphi\left(a_{n} x\right)}\right) x^{2}+\frac{1}{n^{3}} \frac{a_{n}}{n} \frac{\varphi^{(1)}\left(a_{n} x\right)}{\varphi\left(a_{n} x\right)} x\right) \text {. }
\end{aligned}
$$

Remark 5. Taking (2.5) and (2.6) into account, for $x \in[0,+\infty[$ we have

$$
\lim _{n \rightarrow \infty}\left(\left(\frac{a_{n}}{n}\right)^{2} \frac{\varphi^{(2)}\left(a_{n} x\right)}{\varphi\left(a_{n} x\right)}-2 \frac{a_{n}}{n} \frac{\varphi^{(1)}\left(a_{n} x\right)}{\varphi\left(a_{n} x\right)}+1\right)=0 .
$$

In the following, we assume that there exist $\gamma, \delta$, so that $0<\gamma \leq 1, \delta \leq 2, \gamma<\delta$, and the function $\varphi$ verifies the conditions

$$
\begin{gathered}
\lim _{n \rightarrow \infty} n^{\gamma}\left(\left(\frac{a_{n}}{n}\right)^{2} \frac{\varphi^{(2)}\left(a_{n} x\right)}{\varphi\left(a_{n} x\right)}-2 \frac{a_{n}}{n} \frac{\varphi^{(1)}\left(a_{n} x\right)}{\varphi\left(a_{n} x\right)}+1\right)=\beta_{2}(x) \\
\lim _{n \rightarrow \infty} n^{\delta-1}\left(\left(\frac{a_{n}}{n}\right)^{3} \frac{\varphi^{(3)}\left(a_{n} x\right)}{\varphi\left(a_{n} x\right)}-2\left(\frac{a_{n}}{n}\right)^{2} \frac{\varphi^{(2)}\left(a_{n} x\right)}{\varphi\left(a_{n} x\right)}+\frac{a_{n}}{n} \frac{\varphi^{(1)}\left(a_{n} x\right)}{\varphi\left(a_{n} x\right)}\right)=\beta_{3}(x)
\end{gathered}
$$

and

$$
\lim _{n \rightarrow \infty} n^{\delta}\left(\left(\frac{a_{n}}{n}\right)^{4} \frac{\varphi^{(4)}\left(a_{n} x\right)}{\varphi\left(a_{n} x\right)}-4\left(\frac{a_{n}}{n}\right)^{3} \frac{\varphi^{(3)}\left(a_{n} x\right)}{\varphi\left(a_{n} x\right)}+6\left(\frac{a_{n}}{n}\right)^{2} \frac{\varphi^{(2)}\left(a_{n} x\right)}{\varphi\left(a_{n} x\right)}\right.
$$




$$
\left.-4 \frac{a_{n}}{n} \frac{\varphi^{(1)}\left(a_{n} x\right)}{\varphi\left(a_{n} x\right)}+1\right)=\beta_{4}(x),
$$

for any $x \in\left[0,+\infty\left[\right.\right.$, where $\beta_{2}, \beta_{3}, \beta_{4}$ are functions, $\beta_{2}, \beta_{3}, \beta_{4}:[0,+\infty[\rightarrow \mathbb{R}$.

Lemma 5. For any $x \in[0,+\infty[$, the following identities

$$
\begin{gathered}
\lim _{n \rightarrow \infty}\left(T_{n, 0}^{*} \varphi S_{n}\right)(x)=1, \\
\lim _{n \rightarrow \infty} \frac{\left(T_{n, 2}^{*} \varphi S_{n}\right)(x)}{n^{2-\gamma}}=\beta_{2}(x) \cdot x^{2}+\epsilon(\gamma) \cdot x, \\
\lim _{n \rightarrow \infty} \frac{\left(T_{n, 4}^{*} \varphi S_{n}\right)(x)}{n^{4-\delta}}=\beta_{4}(x) \cdot x^{4}+6 \beta_{3}(x) \cdot x^{3}+3 \eta(\delta) \cdot x^{2}
\end{gathered}
$$

hold and there exists $n_{0} \in \mathbb{N}$, so that

$$
\begin{gathered}
\left(T_{n, 0}^{*} \varphi S_{n}\right)(x)=1=k_{0}, \\
\frac{\left(T_{n, 2}^{*} \varphi S_{n}\right)(x)}{n^{2-\gamma}} \leq m_{2}(K) \cdot b^{2}+b+1=k_{2}, \\
\frac{\left(T_{n, 4}^{*} \varphi S_{n}\right)(x)}{n^{4-\delta}} \leq m_{4}(K) \cdot b^{4}+6 m_{3}(K) \cdot b^{3}+3 b^{2}+1=k_{4},
\end{gathered}
$$

for any $x \in K=[0, b], b>0$, any $n \in \mathbb{N}, n \geq n_{0}$ and $m_{2}(K):=\sup _{x \in K}\left|\beta_{2}(x)\right|$,

$m_{3}(K):=\sup _{x \in K}\left|\beta_{3}(x)\right|, m_{4}(K):=\sup _{x \in K}\left|\beta_{4}(x)\right|$, where $\epsilon(\gamma)=\left\{\begin{array}{l}1, \gamma=1 \\ 0,0<\gamma<1\end{array}\right.$ and $\eta(\delta)=\left\{\begin{array}{l}1, \delta=2 \\ 0, \delta<2\end{array}\right.$

Proof. The identities (3.11)-(3.13) follow from Lemma 4, while (3.14)-(3.16) follow from (3.11)-(3.13) by taking the definition of the limit into account.

Let us assume that $I=J=\left[0,+\infty\left[, E(I)=C_{2}([0,+\infty[), F(J)=C([0,+\infty[)\right.\right.$, the functions $\varphi_{n, k}:\left[0,+\infty\left[\rightarrow \mathbb{R}\right.\right.$ let be defined by $\varphi_{n, k}(x)=\frac{1}{\varphi\left(a_{n} x\right)} \frac{\varphi^{(k)}(0)}{k !}\left(a_{n} x\right)^{k}$, for any $x \in\left[0,+\infty\left[\right.\right.$, any $n, k \in \mathbb{N}_{0}, n \neq 0$ and the functionals $A_{n, k}: C_{2}([0,+\infty[) \rightarrow$ $\mathbb{R}$ let be defined by $A_{n, k}(f)=f\left(\frac{k}{n}\right)$, for any $n, k \in \mathbb{N}_{0}, n \neq 0$. In this way we get the $\varphi$-Szász-Mirakjan type operators. 
Theorem 2. Let the function $f \in E(I)$ be given. If $x \in[0,+\infty[, f$ is $s$ times differentiable in a neighborhood of $x$, then:

$$
\lim _{n \rightarrow \infty}\left(\varphi S_{n} f\right)(x)=f(x)
$$

if $s=0$ and

$$
\begin{gathered}
\lim _{n \rightarrow \infty} n^{\gamma}\left(\left(\varphi S_{n} f\right)(x)-f(x)-\left(\frac{a_{n}}{n} \frac{\varphi^{(1)}\left(a_{n} x\right)}{\varphi\left(a_{n} x\right)}-1\right) x f^{(1)}(x)\right) \\
=\frac{1}{2}\left(\beta_{2}(x) \cdot x^{2}+\epsilon(\gamma) \cdot x\right) f^{(2)}(x),
\end{gathered}
$$

if $s=2$.

If $f$ is $s$ times differentiable on $[0,+\infty[$, then the convergence from (3.17) and (3.18) is uniform on any compact interval $K=[0, b] \subset[0,+\infty[$.

Moreover, we get

$$
\left|\left(\varphi S_{n} f\right)(x)-f(x)\right| \leq\left(1+k_{2}\right) \omega_{1}\left(f ; \frac{1}{\sqrt{n^{\gamma}}}\right),
$$

for any $f \in C_{2}\left(\left[0,+\infty[)\right.\right.$, any $x \in K$ and $n \in \mathbb{N}, n \geq n_{0}$.

Proof. It follows from Theorem 1, with $\alpha_{0}=0, \alpha_{2}=2-\gamma$ and $\alpha_{4}=4-\delta$, Lemma 4 and Lemma 5.

Remark 6. If $a_{n}=n$, for any $n \in \mathbb{N}$ and $\varphi(x)=e^{x}$, for any $x \in[0,+\infty[$ we get the well-known results for the classical Szász-Mirakjan operators. In this case $\gamma=1$, $\delta=2, \beta_{2}(x)=\beta_{3}(x)=\beta_{4}(x)=0$, for any $x \in\left[0,+\infty\left[\right.\right.$ and $k_{2}=b, k_{4}=3 b^{2}+b$, (see [12]).

\section{OTHER FORM FOR THE SZÁSZ-MIRAKJAN OPERATORS}

In this section, we assume that $c=1$ in the relation (2.7). In [6] or [14], we can find some known results about differentiation of composed functions.

Theorem 3 ([14]). Let $I, J$ be two intervals of real numbers and let $f: I \rightarrow$ $\mathbb{R}, g: J \rightarrow \mathbb{R}$ be two real-valued functions, such that $f(I) \subset J$. If $f$ is $k$ times differentiable on $I$ and $g$ is $k$ times differentiable on $J, k \in \mathbb{N}$, then the function $g \circ f: I \rightarrow \mathbb{R}$ is also $k$ times differentiable on $I$ and the following identity

$$
\begin{gathered}
(g \circ f)^{(k)}(x)=\sum_{i=1}^{k}\left(g^{(i)} \circ f\right)(x) \sum_{\substack{a_{1}+a_{2}+\ldots+a_{k}=i \\
a_{1}+2 a_{2}+\ldots+k a_{k}=k}} \frac{k !}{a_{1} ! a_{2} ! \ldots a_{k} !} \\
\cdot\left(\frac{f^{(1)}(x)}{1 !}\right)^{a_{1}}\left(\frac{f^{(2)}(x)}{2 !}\right)^{a_{2}} \cdot \ldots \cdot\left(\frac{f^{(k)}(x)}{k !}\right)^{a_{k}}
\end{gathered}
$$

holds, for any $x \in I$. 
Theorem 4. If $k \in \mathbb{N}$, then the following identity

$$
\begin{aligned}
\varphi^{(k)}(0)= & \sum_{i=1}^{k} \sum_{\substack{a_{1}+a_{2}+\ldots+a_{k}=i \\
a_{1}+2 a_{2}+\ldots+k a_{k}=k}} \frac{k !}{a_{1} ! a_{2} ! \ldots a_{k} !}\left(\frac{1+\alpha(0)}{1 !}\right)^{a_{1}} \\
& \cdot\left(\frac{\alpha^{(1)}(0)}{2 !}\right)^{a_{2}} \ldots \cdot\left(\frac{\alpha^{(k-1)}(0)}{k !}\right)^{a_{k}}
\end{aligned}
$$

holds.

Proof. One applies Theorem 3, with $g(x)=e^{x}$, for $x \in \mathbb{R}$ and $f(x)=x+\int_{0}^{x} \alpha(t) d t$, for $x \in[0,+\infty[$.

Remark 7. The relation (3.1) represents the form of $\varphi$-Szász-Mirakjan type operators, by using the function $\varphi$. By replacing the form of $\varphi^{(k)}(0)$, from the relation (4.2) in (3.1), we get the form of the $\varphi$-Szász-Mirakjan type operators, where appears the function $\alpha$.

Now we can give some applications.

Example 1. If $a_{n}=n, n \in \mathbb{N}$ and $\alpha(x)=0$, for any $x \in[0,+\infty[$, from (4.2) follows that $\varphi^{(k)}(0)=1$, for any $k \in \mathbb{N}$. In this case, we get the classical SzászMirakjan operators.

Example 2. Suppose that $\alpha(x)=\frac{1}{(x+1)^{2}}$, for any $x \in\left[0,+\infty\left[\right.\right.$. Then $\alpha^{(p)}(x)=$ $\frac{(-1)^{p}(p+1) !}{(x+1)^{p+2}}, \varphi(x)=e^{x+1-\frac{1}{x+1}}$, for any $x \in\left[0,+\infty\left[\right.\right.$, any $p \in \mathbb{N}_{0}$ and we get

$$
\varphi^{(k)}(0)=\sum_{i=1}^{k} \sum_{\substack{a_{1}+a_{2}+\ldots+a_{k}=i \\ a_{1}+2 a_{2}+\ldots+k a_{k}=k}} \frac{k !}{a_{1} ! a_{2} ! \ldots a_{k} !} 2^{a_{1}}(-1)^{1 a_{2}+2 a_{3}+\ldots+(k-1) a_{k}} .
$$

Taking into account $a_{1}+2 a_{2}+\ldots+k a_{k}=k$ and $a_{1}+a_{2}+\ldots+a_{k}=i$, we get

$$
\varphi^{(k)}(0)=\sum_{i=1}^{k} \sum_{\substack{a_{1}+a_{2}+\ldots+a_{k}=i \\ a_{1}+2 a_{2}+\ldots+k a_{k}=k}} \frac{k !}{a_{1} ! a_{2} ! \ldots a_{k} !}(-1)^{k-i} 2^{a_{1}},
$$

from where, it follows

$$
\varphi^{(k)}(0)=(-1)^{k} \sum_{i=1}^{k} \sum_{\substack{a_{1}+a_{2}+\ldots+a_{k}=i \\ a_{1}+2 a_{2}+\ldots+k a_{k}=k}}(-1)^{i} \frac{k !}{a_{1} ! a_{2} ! \ldots a_{k} !} 2^{a_{1}} .
$$


If we consider $a_{n}=n+\frac{1}{n}$, for any $n \in \mathbb{N}$, the operators defined by (3.1) become

$$
\begin{gathered}
\left(\varphi S_{n} f\right)(x)=e^{\frac{1}{\left(n+\frac{1}{n}\right) x+1}-\left(n+\frac{1}{n}\right) x-1} \sum_{k=0}^{\infty} \frac{(-1)^{k} \sum_{i=1}^{k} \sum_{\begin{array}{c}
a_{1}+a_{2}+\ldots+a_{k}=i \\
a_{1}+2 a_{2}+\ldots+k a_{k}=k
\end{array}}(-1)^{i} \frac{k !}{a_{1} ! a_{2} ! \ldots a_{k} !} 2^{a_{1}}}{k !} \\
\cdot\left(\left(n+\frac{1}{n}\right) x\right)^{k} f\left(\frac{k}{n}\right),
\end{gathered}
$$

for any $f \in C_{2}([0,+\infty[), x \in[0,+\infty[$ and $n \in \mathbb{N}$.

\section{ACKNOWLEDGEMENT}

We thank the reviewer for his suggestions leading to the improvement of the first version of this paper.

\section{REFERENCES}

[1] O. Agratini, Approximation by linear operators. Cluj-Napoca: Presa Universitară Clujeană, 2000.

[2] F. Altomare and M. Campiti, Korovkin-type approximation theory and its applications, ser. de Gruyter Studies in Mathematics. Berlin: Walter de Gruyter, 1994, vol. 17.

[3] D. Bărbosu, O. T. Pop, and D. Miclăuş, "The Kantorovich form of some extensions for SzászMirakjan operators," Rev. Anal. Numér. Théor. Approx., vol. 39, no. 1, pp. 8-20, 2010.

[4] D. Bărbosu, O. T. Pop, and D. Miclăuş, "On some extensions for the Szász-Mirakjan operators," An. Univ. Oradea, Fasc. Mat., vol. 18, pp. 179-187, 2011.

[5] J. Favard, "Sur le multiplicateurs d'interpolation," J. Math. Pures Appl., IX. Sér., vol. 23, pp. 219-247, 1944.

[6] I. S. Gradshteyn and I. M. Ryzhik, Table of integrals, series, and products. New York-LondonToronto: Academic Press (Harcourt Brace Jovanovich, Publishers), 1980.

[7] A. Jakimovski and D. Leviatan, "Generalized Szász operators for the approximation in the finite interval," Mathematica, Cluj, vol. 11(34), pp. 97-103, 1969.

[8] D. Miclăuş and O. T. Pop, "The Voronovskaja theorem for some linear positive operators defined by infinite sum," Creat. Math. \& Inform., vol. 20, no. 1, pp. 55-61, 2011.

[9] D. Miclăuş, O. T. Pop, and D. Bărbosu, "The Voronovskaja type theorem for an extension of Kantorovich operators," An. Univ. Craiova, Ser. Mat. Inf., vol. 37, no. 4, pp. 29-36, 2010.

[10] G. M. Mirakjan, "Approximation of continuous functions with the aid of polynomials," Dokl. Acad. Nauk. SSSR, vol. 31, pp. 201-205, 1941.

[11] C. Mortici, "An extension of the Szász-Mirakjan operators," An. Ştiinţ. Univ. "Ovidius" Constanţa, Ser. Mat., vol. 17, no. 1, pp. 137-144, 2009.

[12] O. T. Pop, "About some linear and positive operators defined by infinite sum," Demonstr. Math., vol. 39, no. 2, pp. 377-388, 2006.

[13] O. T. Pop, D. Bărbosu, and M. Dan, "The Voronovskaja type theorem for an extension of SzászMirakjan operators," Demonstr. Math., vol. 45, no. 1, pp. 107-115, 2012.

[14] I. Păvăloiu and N. Pop, Interpolation and applications (Interpolare şi aplicaţii). Cluj-Napoca: Editura Risoprint, 2005.

[15] O. Szász, "Generalization of S. N. Bernstein's polynomials to the infinite interval," J. Research, National Bureau of Standards, vol. 45, pp. 239-245, 1950. 
Authors' addresses

Ovidiu T. Pop

National College Mihai Eminescu, Mihai Eminescu 5, 440014 Satu Mare, Romania

E-mail address: ovidiutiberiudyahoo.com

Dan Miclăuş

North University of Baia Mare, Department of Mathematics and Computer Science, Victoriei 76, 430122 Baia Mare, Romania

E-mail address: danmiclausrz@yahoo.com

Dan Bărbosu

North University of Baia Mare, Department of Mathematics and Computer Science, Victoriei 76, 430122 Baia Mare, Romania

E-mail address: barbosudan@yahoo.com 